\title{
Solution of Single and Multiobjective Stochastic Inventory Models with Fuzzy Cost Components by Intuitionistic Fuzzy Optimization Technique
}

\author{
S. Banerjee and T. K. Roy \\ Department of Mathematics, Bengal Engineering and Science University, Shibpur, Howrah 711103, India
}

Correspondence should be addressed to S. Banerjee, sou_b@rediffmail.com

Received 14 August 2009; Revised 26 January 2010; Accepted 5 March 2010

Academic Editor: Ching Jong Liao

Copyright (C) 2010 S. Banerjee and T. K. Roy. This is an open access article distributed under the Creative Commons Attribution License, which permits unrestricted use, distribution, and reproduction in any medium, provided the original work is properly cited.

Paknejad et al.'s model is considered in this paper. Itemwise multiobjective models for both exponential and uniform lead-time demand are taken and the results are compared numerically both in fuzzy optimization and intuitionistic fuzzy optimization techniques. Objective of this paper is to establish that intuitionistic fuzzy optimizaion method is better than usual fuzzy optimization technique as expected annual cost of this inventory model is more minimized in case of intuitionistic fuzzy optimization method. As a single objective stochastic inventory model where the lead-time demand follows normal distribution and with varying defective rate, expected annual cost is also measured. Finally the model considers for fuzzy cost components, which make the model more realistic, and numerical values for uniform, exponential, and normal leadtime demand are compared. Necessary graphical presentations are also given besides numerical illustrations.

\section{Introduction}

In conventional inventory models, uncertainties are treated as randomness and are handled by appealing to probability theory. However, in certain situations uncertainties are due to fuzziness and in these cases the fuzzy set theory, originally introduced by Zadeh [1], is applicable. Today most of the real-world decision-making problems in economic, technical and environmental ones are multidimensional and multiobjective. It is significant to realize that multiple-objectives are often noncommensurable and conflict with each other in optimization problem. An objective within exact target value is termed as fuzzy goal. So a multiobjective model with fuzzy objectives is more realistic than deterministic of it.

In decision making process, first, Bellman and Zadeh [2] introduced fuzzy set theory; Tanaka et al. [3] applied concept of fuzzy sets to decision-making problems to consider the objectives as fuzzy goals over the $\alpha$-cuts of a fuzzy constraints. Zimmermann [4, 5] showed 
that the classical algorithms can be used in few inventory models. Li et al. [6] discussed fuzzy models for single-period inventory problem in 2002. Abou-El-Ata et al. [7] considered in 2003 a probabilistic multiitem inventory model with varying order cost. A single-period inventory model with fuzzy demand is analyzed by Kao and Hsu [8]. Fergany and El-Wakeel [9] considered a probabilistic single-item inventory problem with varying order cost under two linear constraints. A survey of literature on continuously deteriorating inventory models are discussed by Raafat [10]. Hala and EI-Saadani [11] analyzed a constrained single period stochastic uniform inventory model with continuous distributions of demand and varying holding cost. Some inventory problems with fuzzy shortage cost is discussed by Katagiri and Ishii [12]. Moon and Choi [13] implemented a note on lead time and distributional assumptions in continuous review inventory models. Lai and Hwang [14, 15] elaborately discussed fuzzy mathematical programming and fuzzy multiple objective decision making in their two renowned contributions. Ouyang and Chang [16] analyzed a minimax distribution free procedure for mixed inventory models involving variable lead time with fuzzy lost sales. Mahapatra and Roy [17] discussed fuzzy multiobjective mathematical programming on reliability optimization model. Hariga and Ben-Daya [18] considered some stochastic inventory models with deterministic variable lead time. A fuzzy EOQ model with demanddependent unit cost under limited storage capacity is implemented by Roy and Maiti [19]. Zheng [20] discussed optimal control policy for stochastic inventory systems with Markovian discount opportunities.

Intuitionistic Fuzzy Set (IFS) was introduced by Atanassov [21] and seems to be applicable to real world problems. The concept of IFS can be viewed as an alternative approach to define a fuzzy set in case where available information is not sufficient for the definition of an imprecise concept by means of a conventional fuzzy set. Thus it is expected that IFS can be used to simulate human decision-making process and any activitities requiring human expertise and knowledge that are inevitably imprecise or totally reliable. Here the degrees of rejection and satisfaction are considered so that the sum of both values is always less than unity [21]. Atanassov also analyzed Intuitionistic fuzzy sets in a more explicit way. Atanassov and Gargov [22] discussed an Open problem in intuitionistic fuzzy sets theory. An Interval-valued intuitionistic fuzzy set was analyzed byAtanassov [23]. Atanassov and Kreinovich [24] implemented Intuitionistic fuzzy interpretation of interval data. The temporal intuitionistic fuzzy sets are discussed also by Atanassov [25]. Intuitionistic fuzzy soft sets are considered by Maji et al. [26]. Nikolova et al. [27] presented a Survey of the research on intuitionistic fuzzy sets. Rough intuitionistic fuzzy sets are analyzed by Rizvi et al. [28]. Angelov [29] implemented the Optimization in an intuitionistic fuzzy environment. He [30, 31] also contributed in another two important papers, based on Intuitionistic fuzzy optimization. Pramanik and Roy [32] solved a vector optimization problem using an Intuitionistic Fuzzy goal programming. A transportation model is solved by Jana and Roy [33] using multi-objective intuitionistic fuzzy linear programming.

Paknejad et al.[35] presented a quality adjusted lot-sizing model with stochastic demand and constant lead time and studied the benefits of lower setup cost in the model. We note that the previous literature focuses on the issue of setup cost reduction in which information about lead-time demand, whether constant or stochastic, is assumed completely known. Ouyang and Chang [34] modified Paknejad et al.'s inventory model by relaxing the assumption that the stochastic demand during lead time follows a specific probability distribution and by considering that the unsatisfied demands are partially backordered. Also, instead of having a stockout cost in the objective function, a service level constraint is employed. 
Paknejad et al.'s [35] model is considered in this paper, as a single objective stochastic inventory model where the lead-time demand follows normal distribution and with varying defective rate, expected annual cost is measured. Itemwise multiobjective models for both exponential and uniform lead time demand are taken and the results are compared numerically both in fuzzy optimization and intuitionistic fuzzy optimization techniques. From our numerical as well as graphical presentations, it is clear that intuitionistic fuzzy optimization obtains better results than fuzzy optimization. Finally the model considers for several fuzzy costs and numerical values for uniform, exponential, and normal lead-time demand are compared. Necessary graphical presentations are also given besides numerical illustrations.

\section{Mathematical Model}

Paknejad et al. [35] presented a quality adjusted lot-sizing model with stochastic demand and constant lead time and studied the benefits of lower setup cost in the model. We note that the previous literature focuses on the issue of setup cost reduction in which information about lead-time demand, whether constant or stochastic, is assumed completely known. This paper considers Paknejad et al.'s model along with the notations and some assumptions that will be taken into account throughout the paper. Each lot contains a random number of defectives following binomial distribution. After the arrival purchaser examines the entire lot, an order of size $Q$ is placed as soon as the inventory position reaches the reorder point $s$. The shortages are allowed and completely backordered. Lead-time is constant and probability distribution of lead-time demand is known.

Now, we use the following notations:

$D$ : expected demand per year,

$Q$ : lot size,

$s$ : reorder point,

$K$ : setup cost,

$\theta$ : defective rate in a lot of size $Q, 0 \leq \theta \leq 1$,

$h$ : nondefective holding cost per unit per year,

$h^{\prime}$ : defective holding cost per unit per year,

$\pi$ : shortage cost per unit short,

$v$ : cost of inspecting a single item in each lot,

$\mu$ : expected demand during lead time,

$\bar{b}(s)$ : the expected demand short at the end of the cycle

$$
\bar{b}(s)=\int_{s}^{\infty}(x-s) f(x) d x,
$$

where $f(x)$ is the density function of lead-time demand,

$\mathrm{EC}(Q, s)$ : expected annual cost given that a lot size $Q$ is ordered. 


\section{Single Objective Stochastic Inventory Model (SOSIM)}

Thus a quality-adjusted lot-sizing model is formed as

$$
\begin{aligned}
\operatorname{MinEC}(Q, s)= & \text { Setup cost }+ \text { non-defective item holding cost }+ \text { stockout cost } \\
& + \text { defective item holding cost }+ \text { inspecting cost } \\
= & \frac{D K}{Q(1-\theta)}+h\left(s-\mu+\frac{1}{2}(Q(1-\theta)+\theta)\right) \\
& +\frac{D \pi \bar{b}(s)}{Q(1-\theta)}+h^{\prime} \theta(Q-1)+\frac{D v}{1-\theta} \quad Q, s>0 .
\end{aligned}
$$

It is the stochastic model, which minimizes the expected annual cost.

\section{Multiobjective Stochastic Inventory Model (MOSIM)}

In reality, a managerial problem of a responsible organization involves several conflicting objectives to be achieved simultaneously that refers to a situation on which the DM has no control. For this purpose a latest tool is linear or nonlinear programming problem with multiple conflicting objectives. So the following model may be considered.

To solve the problem in (3.1) as an MOSIM, it can be reformulated as

$$
\begin{aligned}
\operatorname{MinEC}_{i}\left(Q_{i}, s_{i}\right)= & \frac{D_{i} K_{i}}{Q_{i}\left(1-\theta_{i}\right)}+h_{i}\left(s_{i}-\mu_{i}+\frac{1}{2}\left(Q_{i}\left(1-\theta_{i}\right)+\theta_{i}\right)\right) \\
& +\frac{D_{i} \pi \bar{b}_{i}\left(s_{i}\right)}{Q_{i}\left(1-\theta_{i}\right)}+h_{i}^{\prime} \theta_{i}\left(Q_{i}-1\right)+\frac{D_{i} v_{i}}{1-\theta_{i}} \quad Q_{i}, s_{i}>0 \forall i=1,2, \ldots, n .
\end{aligned}
$$

\section{Multiitem Stochastic Model with Fuzzy Cost Components}

Stochastic nonlinear programming problem with fuzzy cost components considers as

$$
\begin{array}{r}
\operatorname{MinEC}\left(Q_{1}, \ldots, Q_{n}, s_{1}, \ldots, s_{n}\right) \\
=\sum_{i=1}^{n}\left(\frac{D_{i} \tilde{K}_{i}}{Q_{i}\left(1-\theta_{i}\right)}+\widetilde{h}_{i}\left(s_{i}-\mu_{i}+\frac{1}{2}\left(Q_{i}\left(1-\theta_{i}\right)+\theta_{i}\right)\right)+\frac{D_{i} \tilde{\pi}_{i} \bar{b}_{i}(s)}{Q_{i}\left(1-\theta_{i}\right)}+\widetilde{h}_{i}^{\prime} \theta_{i}\left(Q_{i}-1\right)+\frac{D_{i} v_{i}}{1-\theta_{i}}\right) \\
Q_{i}, s_{i}>0 \quad \forall i=1,2, \ldots, n .
\end{array}
$$

Here $\tilde{K}_{i}, \tilde{\pi}_{i}, \tilde{h}_{i}, \tilde{h}_{i}^{\prime}$ represent vector of fuzzy parameters involved in the objective function EC. We assume $\tilde{K}_{i}=\left(K_{i}^{-}, K_{i}^{0}, K_{i}^{+}\right), \tilde{\pi}_{i}=\left(\pi_{i}^{-}, \pi_{i}^{0}, \pi_{i}^{+}\right), \tilde{h}_{i}=\left(h_{i}^{-}, h_{i}^{0}, h_{i}^{+}\right)$, and $\tilde{h}_{i}^{\prime}=\left(h_{i}^{\prime-}, h_{i}^{\prime 0}, h_{i}^{\prime+}\right)$, all of which are triangular fuzzy numbers. 


\section{Fuzzy Nonlinear Programming (FNLP) Technique to Solve Multiobjective Nonlinear Programming Problem (MONLP)}

A Multi-Objective Non-Linear Programming (MONLP) or Vector Minimization problem (VMP) may be taken in the following form:

$$
\begin{aligned}
& \qquad \operatorname{Minf}(x)=\left(f_{1}(x), f_{2}(x), \ldots, f_{k}(x)\right)^{T} \\
& \text { Subject to } x \in X=\left\{x \in R^{n}: g_{j}(x) \leq \text { or }=\text { or } \geq b_{j} \text { for } j=1,2, \ldots, m\right\} \\
& \qquad l_{i} \leq x \leq u_{i} \quad(i=1,2, \ldots, n) .
\end{aligned}
$$

Zimmermann [5] showed that fuzzy programming technique can be used to solve the multiobjective programming problem.

To solve MONLP problem, the following steps are used.

Step 1. Solve the MONLP of (6.1) as a single objective non-linear programming problem using only one objective at a time and ignoring the others; these solutions are known as ideal solution.

Step 2. From the result of Step 1, determine the corresponding values for every objective at each solution derived. With the values of all objectives at each ideal solution, pay-off matrix can be formulated as follows:

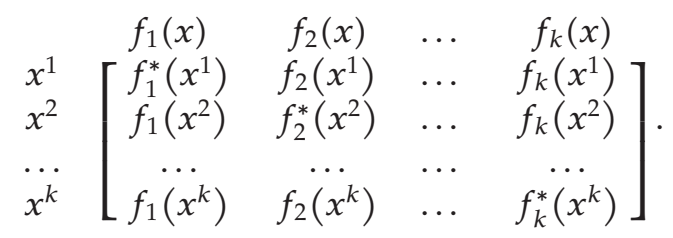

Here $x^{1}, x^{2}, \ldots, x^{k}$ are the ideal solutions of the objective functions $f_{1}(x), f_{2}(x), \ldots, f_{k}(x)$, respectively.

So

$$
\begin{aligned}
U_{r} & =\max \left\{f_{r}\left(x_{1}\right), f_{r}\left(x_{2}\right), \ldots, f_{r}\left(x_{k}\right)\right\}, \\
L_{r} & =\min \left\{f_{r}\left(x_{1}\right), f_{r}\left(x_{2}\right), \ldots, f_{r}\left(x_{k}\right)\right\}
\end{aligned}
$$

( $L_{r}$ and $U_{r}$ are lower and upper bounds of the $r$ th objective functions $\left.f_{r}(x) r=1,2, \ldots, k\right)$ ).

Step 3. Using aspiration level of each objective of the MONLP of (6.1) may be written as follows.

Find $x$ so as to satisfy

$$
f_{r}(x) \tilde{\leq} L_{r} \quad(r=1,2, \ldots, k), x \in X
$$


Here objective functions of (6.1) are considered as fuzzy constraints. These type of fuzzy constraints can be quantified by eliciting a corresponding membership function:

$$
\mu_{r}\left(f_{r}(x)\right)= \begin{cases}0 \text { or } \longrightarrow 0 & \text { if } f_{r}(x) \geq U_{r} \\ \mu_{r}\left(f_{r}(x)\right) & \text { if } L_{r} \leq f_{r}(x) \leq U_{r}(r=1,2, \ldots, k) \\ 1 & \text { if } f_{r}(x) \leq L_{r} .\end{cases}
$$

Having elicited the membership functions (as in (6.5)) $\mu_{r}\left(f_{r}(x)\right)$ for $r=1,2, \ldots, k$ introduce a general aggregation function:

$$
\mu_{\tilde{D}}(x)=G\left(\mu_{1}\left(f_{1}(x)\right), \mu_{2}\left(f_{2}(x)\right), \ldots, \mu_{k}\left(f_{k}(x)\right)\right) .
$$

So a fuzzy multi-objective decision making problem can be defined as

$$
\begin{array}{rr}
\operatorname{Max} & \mu_{\tilde{D}}(x) \\
\text { subject to } & x \in X .
\end{array}
$$

Here we adopt the fuzzy decision as follows.

Fuzzy decision is based on minimum operator (like Zimmermann's approach [4]). In this case (6.7) is known as FNLP $_{\mathrm{M}}$.

Then the problem of (6.7), using the membership function as in (6.5), according to min-operator is reduced to

$$
\begin{aligned}
\operatorname{Max} & \alpha \\
\text { Subject to } & \mu_{i}\left(f_{i}(x)\right) \geq \alpha \text { for }(i=1,2, \ldots, k), x \in X \quad \alpha \in[0,1] .
\end{aligned}
$$

Step 4. Solve (6.8) to get optimal solution.

\section{Stochastic Models with Fuzzy Cost Components}

Stochastic non-linear programming problem with fuzzy objective coefficient considers as

$$
\operatorname{Min} Z=\tilde{C} X, \quad X \geq 0
$$


Here $\tilde{C}$ represents a vector of fuzzy parameters involved in the objective function $Z$. We assume $\widetilde{C}_{i}=\left(c_{i}{ }^{-}, c_{i}{ }^{0}, c_{i}{ }^{+}\right)$, which is a triangular fuzzy number with membership function:

$$
\mu_{\widetilde{C}_{i}}(t)= \begin{cases}\frac{t-c_{i}^{-}}{c_{i}^{0}-c_{i}^{-}} & \text {for } c_{i}^{-} \leq t \leq c_{i}^{0}, \\ \frac{c_{i}^{+}-t}{c_{i}^{+}-c_{i}^{0}} & \text { for } c_{i}^{0} \leq t \leq c_{i}^{+}, \\ 0 & \text { for } t>c_{i}^{+} \text {or } t<c_{i}^{-} .\end{cases}
$$

So (7.1) becomes

$$
\operatorname{Min} Z=\left(c_{i}^{-} X, c_{i}^{0} X, c_{i}^{+} X\right), \quad X \geq 0,
$$

where

$$
\begin{aligned}
& c^{-}=\left(c_{1}^{-}, c_{2}^{-}, \ldots, c_{n}^{-}\right), \\
& c^{0}=\left(c_{1}^{0}, c_{2}^{0}, \ldots, c_{n}^{0}\right), \\
& c^{+}=\left(c_{1}^{+}, c_{2}^{-}, \ldots, c_{n}^{+}\right) .
\end{aligned}
$$

According to Kaufman and Gupta [36] by combining three objectives into a single objective function, (7.3) can be reduced to an LPP by most likely criteria as

$$
\operatorname{Min}\left(\frac{c^{-}+4 c^{0}+c^{+}}{6}\right) X, \quad X \geq 0
$$

\section{Formulation of Intuitionistic Fuzzy Optimization}

When the degree of rejection (nonmembership) is defined simultaneously with degree of acceptance (membership) of the objectives and when both of these degrees are not complementary to each other, then IF sets can be used as a more general tool for describing uncertainty. 


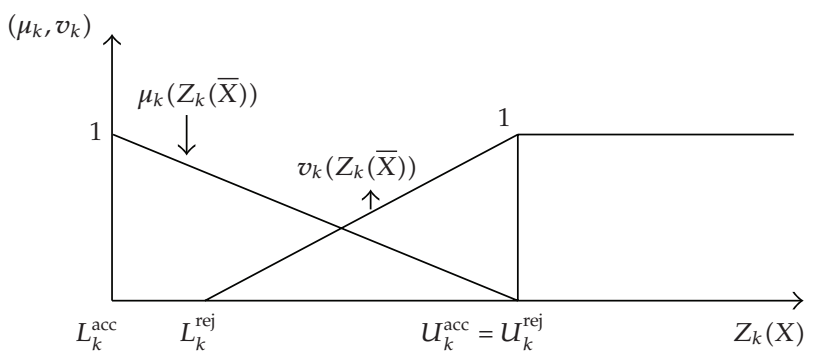

Figure 1: Membership and nonmemebership functions of the objective goal.

To maximize the degree of acceptance of IF objectives and constraints and to minimize the degree of rejection of IF objectives and constraints, we can write

$$
\begin{aligned}
\max & \mu_{i}(\bar{X}), \quad \bar{X} \varepsilon R, \quad i=1,2, \ldots, K+n, \\
\min & v_{i}(\bar{X}), \quad \bar{X} \varepsilon R, \quad i=1,2, \ldots, K+n, \\
\text { Subject to } & v_{i}(\bar{X}) \geq 0 \\
& \mu_{i}(\bar{X}) \geq v_{i}(\bar{X}) \\
& \mu_{i}(\bar{X})+v_{i}(\bar{X})<1 \\
& \bar{X} \geq 0
\end{aligned}
$$

where $\mu_{i}(\bar{X})$ denotes the degree of membership function of $(\bar{X})$ to the ith IF sets and $v_{i}(\bar{X})$ denotes the degree of nonmembership (rejection) of $(\bar{X})$ from the $i$ th IF sets.

\section{An Intuitionistic Fuzzy Approach for Solving MOIP with Linear Membership and Nonmembership Functions}

To define the membership function of MOIM problem, let $L_{k}^{\text {acc }}$ and $U_{k}^{\text {acc }}$ be the lower and upper bounds of the $k$ th objective function. These values are determined as follows. Calculate the individual minimum value of each objective function as a single objective IP subject to the given set of constraints. Let $\bar{X}_{1}^{*}, \bar{X}_{2}^{*}, \ldots, \bar{X}_{k}^{*}$ be the respective optimal solution for the $k$ different objective and evaluate each objective function at all these $k$ optimal solutions. It is assumed here that at least two of these solutions are different for which the $k$ th objective function has different bounded values. For each objective, find lower bound (minimum value) $L_{k}^{\text {acc }}$ and the upper bound (maximum value) $U_{k}^{\text {acc }}$. But in intuitionistic fuzzy optimization (IFO), the degree of rejection (non-membership) and degree of acceptance (membership) are considered so that the sum of both values is less than one. To define membership function of MOIM problem, let $L_{k}^{\text {rej }}$ and $U_{k}^{\text {rej }}$ be the lower and upper bounds of the objective function $Z_{k}(\bar{X})$ where $L_{k}^{\text {acc }} \leq L_{k}^{\text {rej }} \leq U_{k}^{\text {rej }} \leq U_{k}^{\text {acc }}$. These values are defined as follows. 
The linear membership function for the objective $Z_{k}(\bar{X})$ is defined as

$$
\begin{aligned}
& \mu_{k}\left(Z_{k}(\bar{X})\right)= \begin{cases}1 & \text { if } Z_{k}(\bar{X}) \leq L_{k}^{\text {acc }}, \\
\frac{U_{k}^{\text {acc }}-Z_{k}(\bar{X})}{U_{k}^{\text {acc }}-L_{k}^{\text {acc }}} & \text { if } L_{k}^{\text {acc }} \leq Z_{k}(\bar{X}) \leq U_{k}^{\text {acc }}, \\
0 & \text { if } Z_{k}(\bar{X}) \geq U_{k}^{\text {acc }},\end{cases} \\
& v_{k}\left(Z_{k}(\bar{X})\right)= \begin{cases}1 & \text { if } Z_{k}(\bar{X}) \geq U_{k}^{\text {rej },} \\
\frac{Z_{k}(\bar{X})-L_{k}^{\text {rej }}}{U_{k}^{\text {rej }}-L_{k}^{\text {rej }}} & \text { if } L_{k}^{\text {rej }} \leq Z_{k}(\bar{X}) \leq U_{k}^{\text {rej }}, \\
0 & \text { if } Z_{k}(\bar{X}) \leq L_{k}^{\text {rej }} .\end{cases}
\end{aligned}
$$

Lemma 9.1. In case of minimization problem, the lower bound for non-membership function (rejection) is always greater than that of the membership function (acceptance). follows:

Now, we take new lower and upper bounds for the non-membership function as

$$
\begin{gathered}
L_{k}^{\mathrm{rej}}=L_{k}^{\mathrm{acc}}+t\left(U_{k}^{\mathrm{acc}}-L_{k}^{\mathrm{acc}}\right), \quad \text { where } 0<t<1, \\
U_{k}^{\mathrm{rej}}=U_{k}^{\mathrm{acc}}+t\left(U_{k}^{\mathrm{acc}}-L_{k}^{\mathrm{acc}}\right) \quad \text { for } t=0 .
\end{gathered}
$$

Following the fuzzy decision of Bellman-Zadeh [2] together with linear membership function and non-membership functions of (9.1), an intuitionistic fuzzy optimization model of MOIM problem can be written as

$$
\begin{aligned}
\max & \mu_{k}(\bar{X}), \quad \bar{X} \varepsilon R, \quad k=1,2, \ldots, K, \\
\min \quad & v_{k}(\bar{X}), \quad \bar{X} \varepsilon R, \quad k=1,2, \ldots, K \\
\text { Subject to } \quad & v_{k}(\bar{X}) \geq 0 \\
& \mu_{k}(\bar{X}) \geq v_{k}(\bar{X}) \\
& \mu_{k}(\bar{X})+v_{k}(\bar{X})<1 \\
& \bar{X} \geq 0
\end{aligned}
$$


The problem of (9.3) can be reduced following Angelov [29] to the following form:

$$
\begin{aligned}
\text { Max } & \alpha-\beta \\
\text { Subject to } & Z_{k}(\bar{X}) \leq U_{k}^{\mathrm{acc}}-\alpha\left(U_{k}^{\mathrm{acc}}-L_{k}^{\mathrm{acc}}\right), \\
& Z_{k}(\bar{X}) \leq L_{k}^{\mathrm{rej}}+\beta\left(U_{k}^{\mathrm{rej}}-L_{k}^{\mathrm{rej}}\right), \\
& \beta \geq 0, \\
& \alpha \geq \beta, \\
& \alpha+\beta<1, \\
& \bar{X} \geq 0,
\end{aligned}
$$

Then the solution of the MOIM problem is summarized in the following steps.

Step 1. Pick the first objective function and solve it as a single objective IP subject to the constraint; continue the process $K$-times for $K$ different objective functions. If all the solutions (i.e., $\left.\bar{X}_{1}^{*}=\bar{X}_{2}^{*}=\cdots=\bar{X}_{k}^{*}(i=1,2, \ldots, m ; j=1,2, \ldots, n)\right)$ are the same, then one of them is the optimal compromise solution and go to Step 6. Otherwise go to Step 2. However, this rarely happens due to the conflicting objective functions.

Then the intuitionistic fuzzy goals take the form

$$
Z_{k}(\bar{X}) \widetilde{\leq} L_{k}(\bar{X})_{k}^{*} \quad k=1,2, \ldots, K
$$

Step 2. To build membership function, goals and tolerances should be determined at first. Using the ideal solutions, obtained in Step 1, we find the values of all the objective functions at each ideal solution and construct pay off matrix as follows:

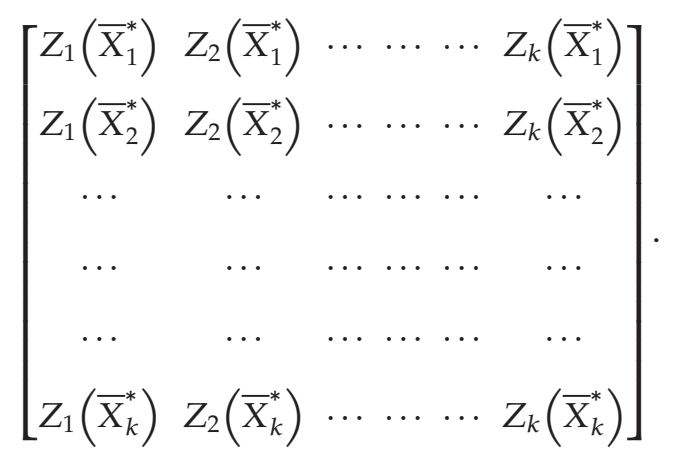

Step 3. From Step 2, we find the upper and lower bounds of each objective for the degree of acceptance and rejection corresponding to the set of solutions as follows:

$$
U_{k}^{\mathrm{acc}}=\max \left(Z_{k}\left(\bar{X}_{r}^{*}\right)\right), \quad L_{k}^{\mathrm{acc}}=\min \left(Z_{k}\left(\bar{X}_{r}^{*}\right)\right), \quad 1 \leq r \leq k .
$$


For linear membership functions,

$$
\begin{gathered}
L_{k}^{\mathrm{rej}}=L_{k}^{\mathrm{acc}}+t\left(U_{k}^{\mathrm{acc}}-L_{k}^{\mathrm{acc}}\right), \quad \text { where } 0<t<1, \\
U_{k}^{\mathrm{rej}}=U_{k}^{\mathrm{acc}}+t\left(U_{k}^{\mathrm{acc}}-L_{k}^{\mathrm{acc}}\right) \quad \text { for } t=0 .
\end{gathered}
$$

Step 4. Construct the fuzzy programming problem of (9.3) and find its equivalent LP problem of (9.4).

Step 5. Solve (9.4) by using appropriate mathematical programming algorithm to get an optimal solution and evaluate the $K$ objective functions at these optimal compromise solutions.

Step 6. STOP.

\section{Few Stochastic Models}

Case 1 (Demand follows Uniform distribution). We assume that lead time demand for the period for the $i$ th item is a random variable which follows uniform distribution and if the decision maker feels that demand values for item $I$ below $a_{i}$ or above $b_{i}$ are highly unlikely and values between $a_{i}$ and $b_{i}$ are equally likely, then the probability density function $f_{i}(x)$ is given by

$$
f_{i}(x)=\left\{\begin{array}{ll}
\frac{1}{b_{i}-a_{i}}, & \text { if } a_{i} \leq x \leq b_{i}, \\
0, & \text { otherwise }
\end{array} \quad \text { for } i=1,2, \ldots, n\right.
$$

So,

$$
\bar{b}_{i}\left(s_{i}\right)=\frac{\left(b_{i}-s_{i}\right)^{2}}{2\left(b_{i}-a_{i}\right)} \quad \text { for } i=1,2, \ldots, n
$$

where $\bar{b}_{i}\left(s_{i}\right)$ are the expected number of shortages per cycle and all these values of $\bar{b}_{i}\left(s_{i}\right)$ affect all the desired models.

Case 2 (Demand follows Exponential distribution). We assume that lead-time demand for the period for the $i$ th item is a random variable that follows exponential distribution. Then the probability density function $f_{i}(x)$ is given by

$$
f_{i}(x)= \begin{cases}\lambda_{i} e^{\left(-\lambda_{i} x\right)}, x>0 & \text { for } i=1,2, \ldots, n, \\ 0, & \text { otherwise. }\end{cases}
$$

So,

$$
\bar{b}_{i}\left(s_{i}\right)=\frac{e^{-\lambda_{i} s_{i}}}{\left(-\lambda_{i}\right)} \quad \text { for } i=1,2, \ldots, n
$$


where $\bar{b}_{i}\left(s_{i}\right)$ are the expected number of shortages per cycle and all these values of $\bar{b}_{i}\left(s_{i}\right)$ affect all the desired models.

Case 3 (Demand follows Normal distribution). We assume that lead-time demand for the period for the $i$ th item is a random variable, which follows normal distribution. Then the probability density function $f_{i}(x)$ is given by

$$
\begin{gathered}
f_{i}(x)=\frac{1}{\sigma_{i} \sqrt{2 \pi}} \exp \left(-\frac{\left(x-\mu_{i}\right)^{2}}{2 \sigma_{i}^{2}}\right), \quad-\infty<x<\infty, \\
\bar{b}_{i}\left(s_{i}\right)=\frac{\left(\mu_{i}-s_{i}\right)}{\sigma_{i} \sqrt{2 \pi}}\left(1-\Phi\left(\frac{s_{i}-\mu_{i}}{\sigma_{i}}\right)\right)+\frac{1}{\sqrt{2 \pi}} \exp \left(-\frac{\left(\mu_{i}-s_{i}\right)^{2}}{2 \sigma_{i}^{2}}\right) \text { for } i=1,2, \ldots, n,
\end{gathered}
$$

where $\bar{b}_{i}\left(s_{i}\right)$ are the expected number of shortages per cycle and all these values of $\bar{b}_{i}\left(s_{i}\right)$ affects all the desired models and $\Phi\left(x_{i}\right)$ represents area under standard normal curve from $-\infty$ to $x_{i}$.

\section{Numericals}

\subsection{Solution of the Model of (3.1)}

The lead-time demand follows normal distribution and thus $\bar{b}_{i}\left(s_{i}\right)$, the expected demand short at the end of the cycle, takes up the value according to (10.2). Thus for single item model, we consider the following data:

$$
D=2750 ; \quad K=10 ; \quad h=0.25 ; \quad v=0.02 ; \quad \pi=1 ; \quad h^{\prime}=0.15
$$

(all the cost-related parameters are measured in " $\$$ ").

Here, the lead-time demand follows Normal distribution with mean $\mu=20$ and standard deviation $\sigma=2$.

The lead-time demand follows normal distribution and thus $\bar{b}_{i}\left(s_{i}\right)$, the expected demand short at the end of the cycle, takes up the value according to (10.2).

In Table 1, a study of expected annual cost $\operatorname{EC}(Q, s)$ with lot size $Q$ and reorder point $\mathrm{s}$ is given for different defective rate $\theta$. We conclude from Table 1 as well asFigure 2 that the order quantity as well as the expected annual cost increases as $\theta$ increases.

\subsection{Solution of the Model of (4.1)}

In case of MOSIM of (4.1), we use the methods described in Section 6, to solve it by fuzzy optimization technique, and Sections 8 and 9 , to solve it by intuitionistic fuzzy optimization technique and the following data are considered. 


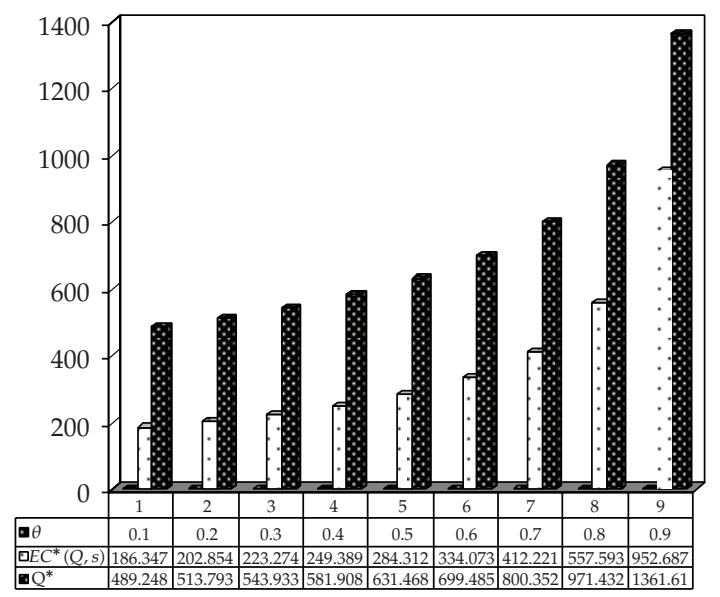

Figure 2

Table 1: Variation of "EC" and "s" with " $\theta$ ".

\begin{tabular}{lcc}
\hline$\theta$ & $\mathrm{EC}^{*}(\$)$ & $\mathrm{Q}^{*}$ \\
\hline 0.1 & 186.3471 & 489.2484 \\
0.2 & 202.8538 & 513.7929 \\
0.3 & 223.2740 & 543.9329 \\
0.4 & 249.3887 & 581.9080 \\
0.5 & 284.3121 & 631.4677 \\
0.6 & 334.0731 & 699.4850 \\
0.7 & 412.2208 & 800.3515 \\
0.8 & 557.5926 & 971.4324 \\
0.9 & 952.6872 & 1361.609 \\
\hline
\end{tabular}

Case 1. The lead-time demand follows uniform distribution and thus $\bar{b}_{i}\left(s_{i}\right)$, the expected demand short at the end of the cycle, takes up the value according to (10.2).

We consider two different sets of data as

$$
\begin{gathered}
D_{1}=2700 ; \quad K_{1}=12 ; \quad h_{1}=0.55 ; \quad \theta_{1}=0.6 ; \quad \mu_{1}=\frac{\left(a_{1}+b_{1}\right)}{2} ; \\
v_{1}=0.03 ; \quad \pi_{1}=1 ; \quad h_{1}^{\prime}=0.25 ; \quad a_{1}=20 ; \quad b_{1}=70 ; \quad \mu_{1}=\frac{\left(a_{1}+b_{1}\right)}{2} . \\
D_{2}=2750 ; \quad K_{2}=10 ; \quad h_{2}=0.25 ; \quad \theta_{2}=0.8 ; \quad \mu_{2}=\frac{\left(a_{2}+b_{2}\right)}{2} ; \quad v_{2}=0.02 ; \\
\pi_{2}=2 ; \quad h_{2}^{\prime}=0.15 ; \quad a_{2}=10 ; \quad b_{2}=50 ; \quad \mu_{2}=\frac{\left(a_{2}+b_{2}\right)}{2} .
\end{gathered}
$$


Table 2: Comparison of solutions of FO and IFO (UNIFORM).

\begin{tabular}{lcccccccc}
\hline Methods & $Q_{1}$ & $Q_{2}$ & $s_{1}$ & $S_{2}$ & $\mathrm{EC}_{1}(\$)$ & $\mathrm{EC}_{2}(\$)$ & $\alpha^{*}$ & $\beta^{*}$ \\
\hline $\begin{array}{l}\text { Fuzzy } \\
\text { optimization }\end{array}$ & 415.3 & 1042.3 & 69.157 & 44.778 & 519.0996 & 570.8203 & 0.9599 & - \\
$\begin{array}{l}\text { Intuitionistic } \\
\text { Fuzzy }\end{array}$ & 967.6 & 1136.8 & 65.371 & 49.647 & 516.8335 & 563.7091 & 0.76 & 0.023 \\
optimization & & & & & & & & \\
\hline
\end{tabular}

The pay-off matrix is

$\mathrm{EC}_{1} \quad \mathrm{EC}_{2}$

$\left[\begin{array}{ll}506.0453 & 804.4216 \\ 831.6330 & 562.4362\end{array}\right]$

We take

$$
\begin{array}{cll}
U_{1}^{\text {acc }}=831.6330 ; \quad L_{1}^{\text {acc }}=506.0453 ; & U_{2}^{\text {acc }}=804.4216 ; & L_{2}^{\text {acc }}=562.4362 ; \\
U_{1}^{\text {rej }}=831.6330 ; \quad L_{1}^{\text {rej }}=507 ; & U_{2}^{\text {rej }}=804.4216 ; & L_{2}^{\text {rej }}=570
\end{array}
$$

(all the cost -related parameters are measured in " $\$$ ").

Then from Table 2 andFigure 3 we conclude that Intuitionistic fuzzy optimization (IFO) obtains more optimized values of $\mathrm{EC}_{1}$ and $\mathrm{EC}_{2}$ than fuzzy optimization (FO). Also solution obtained by IFO $(516.8335,563.7091)$ is closer to the ideal solution $(506.0453$, 562.4362) than the solution obtained by FO.

Case 2. The lead-time demand follows exponential distribution and thus $\bar{b}_{i}\left(s_{i}\right)$, the expected demand short at the end of the cycle, takes up the value according to (10.4).

We consider two different sets of data as

$$
\begin{gathered}
D_{1}=2700 ; \quad K_{1}=8 ; \quad h_{1}=1 ; \quad \theta_{1}=0.4 ; \quad \mu_{1}=\frac{1}{\lambda_{1}} ; \quad v_{1}=0.03 ; \\
\pi_{1}=1 ; \quad h_{1}^{\prime}=0.25 ; \quad \lambda_{1}=1 ; \\
D_{2}=2750 ; \quad K_{2}=10 ; \quad h_{2}=1 ; \quad \theta_{2}=0.7 ; \quad v_{2}=0.02 ; \quad \pi_{2}=1.1 ; \\
h_{2}^{\prime}=0.15 ; \quad \mu_{2}=\frac{1}{\lambda_{2}} ; \quad \lambda_{2}=1.1 .
\end{gathered}
$$

The pay-off matrix is

$\mathrm{EC}_{1} \quad \mathrm{EC}_{2}$

$\left[\begin{array}{ll}378.0060 & 523.5858 \\ 551.0537 & 382.4234\end{array}\right]$ 
Table 3: Comparison of solutions of FO and IFO (EXPONENTIAL).

\begin{tabular}{lcccccccc}
\hline Methods & $Q_{1}$ & $Q_{2}$ & $s_{1}$ & $s_{2}$ & $\mathrm{EC}_{1}(\$)$ & $\mathrm{EC}_{2}(\$)$ & $\alpha^{*}$ & $\alpha *$ \\
\hline $\begin{array}{l}\text { Fuzzy } \\
\text { optimization }\end{array}$ & 341.94 & 941.24 & 1.23 & 2.24 & 381.4218 & 434.5079 & 0.39646 & - \\
$\begin{array}{l}\text { Intuitionistic } \\
\text { fuzzy } \\
\text { optimization }\end{array}$ & 201.73 & 939.16 & 3.10 & 2.28 & 377.5540 & 412.6827 & 0.78564 & 0.1026 \\
\hline
\end{tabular}

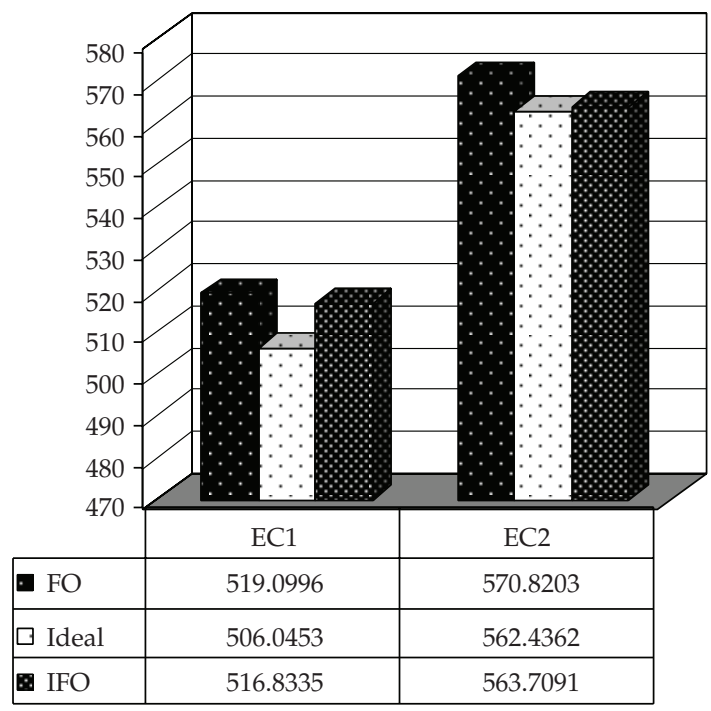

Figure 3

We take

$$
\begin{array}{clll}
U_{1}^{\text {acc }}=551.0537 ; \quad L_{1}^{\text {acc }}=378.0060 ; & U_{2}^{\text {acc }}=523.5858 ; & L_{2}^{\text {acc }}=382.4234 ; \\
U_{1}^{\text {rej }}=551.0537 ; \quad L_{1}^{\text {rej }}=380 ; & U_{2}^{\text {rej }}=523.5858 ; & L_{2}^{\text {rej }}=400 .
\end{array}
$$

(all the cost-related parameters are measured in "\$").

Thus from Table 3 and Figure 4 we conclude that Intuitionistic fuzzy optimization (IFO) obtains more optimized values of $\mathrm{EC}_{1}$ and $\mathrm{EC}_{2}$ than fuzzy optimization (FO). Also solution obtained by IFO $(377.5540,412.6827)$ is closer to the ideal solution (378.0060, 382.4234) than the solution obtained by FO.

Expected annual costs $\mathrm{EC}_{1}$ and $\mathrm{EC}_{2}$ are more minimized in case of IFO than FO because, according to Section 6, only the membership functions $\mu\left(\mathrm{EC}_{1}\right)$ and $\mu\left(\mathrm{EC}_{2}\right)$ are maximized when FO technique is applied and the degree of acceptance of the IF objectives is measured by $\alpha^{*}$. But, when IFO technique is applied, according to Sections 8 and 9 not only are the membership functions $\mu\left(\mathrm{EC}_{1}\right)$ and $\mu\left(\mathrm{EC}_{2}\right)$ maximized but also the non-membership functions $v\left(\mathrm{EC}_{1}\right)$ and $v\left(\mathrm{EC}_{2}\right)$ are minimized and degree of acceptance of the IF objectives is measured by $\alpha^{*}$ as well as degree of rejection of the IF objectives is measured by $\beta^{*}$. As a result of that, IFO obtains more optimized values than FO. 


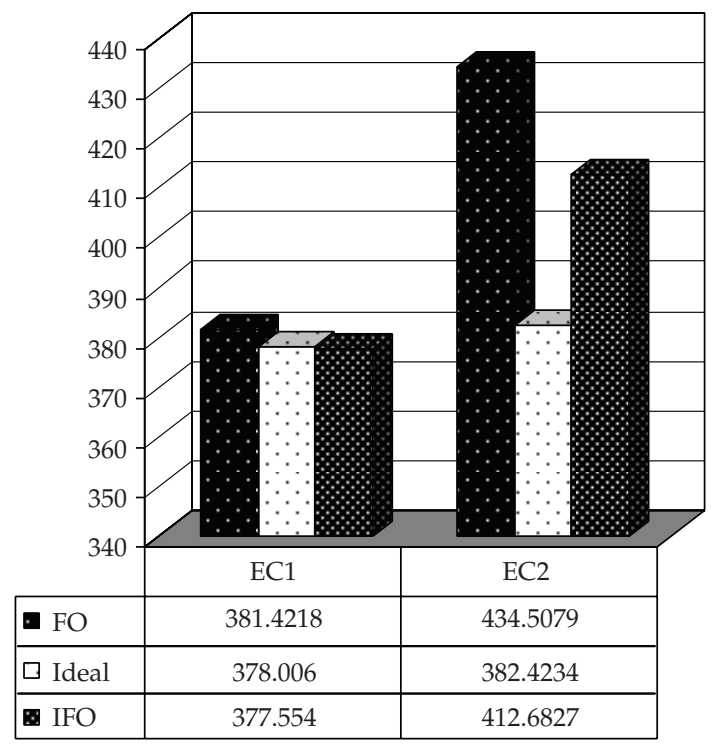

Figure 4

\subsection{Solution of the Model of (5.1)}

In this case our objective is to analyze the expected annual cost of different stochastic models when their cost components are not deterministic but several triangular fuzzy numbers and thus the model becomes more practical and realistic.

To solve the model of (5.1) we use the method described in Section 7.

The lead time demand follows uniform, exponential, and normal distribution, respectively, and thus the expected demand short at the end of the cycle, takes up the value according to (10.2), (10.4), and (10.5), respectively.

In case of Uniform demand,

$$
\begin{gathered}
D_{1}=2750 ; \quad K_{1}=(10,15,20) ; \quad \tilde{h}_{1}=(0.25,0.35,0.45) ; \\
\theta_{1}=0.8 ; \quad \mu_{1}=\frac{\left(a_{1}+b_{1}\right)}{2} ; \quad v_{1}=0.02 ; \\
\tilde{\pi}_{1}=(1,2,3) ; \quad \tilde{h}_{1}^{\prime}=(0.15,0.20,0.25) ; \quad a_{1}=10 ; \\
b_{1}=50 ; \quad p_{1}=3 ; \\
\tilde{K}_{2}=2800 ; \quad \tilde{K}_{2}=(14,16,18) ; \quad \tilde{h}_{2}=(0.65,0.85,1.05) ; \\
\theta_{2}=0.5 ; \quad \mu_{2}=\frac{\left(a_{2}+b_{2}\right)}{2} ; \quad v_{2}=0.03 ; \\
\tilde{\pi}_{1}=(2,3,4) ; \quad \tilde{h}_{2}^{\prime}=(0.45,0.55,0.65) ; \\
a_{2}=15 ; \quad b_{2}=60 ; \quad p_{2}=4 .
\end{gathered}
$$


Table 4: Expected Annual costs of different stochastic models.

\begin{tabular}{lccccc}
\hline Prob. distribution & EC $(\$)$ & $Q_{1}$ & $Q_{2}$ & $s_{1}$ & $s_{2}$ \\
\hline UNIFORM & 1459.05 & 491.64 & 256.26 & 49.74 & 59.41 \\
EXPONENTIAL & 1260.15 & 502.27 & 750.00 & 2.9 & 3.01 \\
NORMAL & 831.05 & 335.5 & 305.79 & 20.86 & 10.55 \\
\hline
\end{tabular}

In case of Exponential demand,

$$
\begin{gathered}
D_{1}=2750 ; \quad \tilde{K}_{1}=(10,12,14) ; \quad \tilde{h}_{1}=(1,2,3) ; \quad \theta_{1}=0.7 ; \quad \lambda_{1}=1 ; \\
v_{1}=0.02 ; \quad \tilde{\pi}_{1}=(1,2,3) ; \quad \tilde{h}_{1}^{\prime}=(0.15,0.20,0.25) ; \quad p_{1}=4 ; \\
D_{2}=2700 ; \quad \tilde{K}_{2}=(8,10,12) ; \quad \tilde{h}_{2}=(0.6,0.8,1) ; \quad \theta_{2}=0.8 ; \quad \lambda_{2}=1.1 ; \quad v_{2}=0.02 ; \\
\tilde{\pi}_{2}=(1,2,3) ; \quad \tilde{h}_{2}^{\prime}=(0.15,0.20,0.25) ; \quad p_{2}=3 .
\end{gathered}
$$

In case of Normal demand,

$$
\begin{array}{ccccc}
D_{1}=2750 ; \quad \tilde{K}_{1}=(10,15,20) ; \quad \tilde{h}_{1}=(0.25,0.3,0.35) ; & \theta_{1}=0.3 ; & \\
v_{1}=0.02 ; \quad \tilde{\pi}_{1}=(1,2,3) ; \quad \tilde{h}_{1}^{\prime}=(0.15,0.25,0.35) ; \quad \mu_{1}=20 ; \quad \sigma_{1}=2 ; \quad p_{1}=2 ; \\
D_{2}=2700 ; \quad \tilde{K}_{2}=(20,25,30) ; \quad \tilde{h}_{2}=(0.45,0.55,0.65) ; \quad \theta_{2}=0.4 ; \quad \lambda_{2}=1.1 ; \\
v_{2}=0.01 ; \quad \tilde{\pi}_{2}=(2,3,4) ; \quad \tilde{h}_{2}^{\prime}=(0.35,0.45,0.55) ; \quad \mu_{2}=10 ; \quad \sigma_{1}=1 ; \quad p_{2}=5
\end{array}
$$

(all the cost-related parameters are measured in " $\$$ ").

\section{Conclusion}

Paknejad et al.'s model is considered in this paper, as a single objective stochastic inventory model where the lead-time demand follows normal distribution, and with varying defective rate, expected annual cost is measured. Our objective is to minimize the expected annual cost. Itemwise multiobjective models for both exponential and uniform lead-time demand are taken and the results are compared numerically both in fuzzy optimization and in intuitionistic fuzzy optimization techniques. From our numerical as well as graphical presentations, it is clear that intuitionistic fuzzy optimization obtains better results than fuzzy optimization. Thus expected annual cost is more minimized in case of intuitionistic fuzzy optimization than the usual fuzzy optimization technique. Finally the model considers for several fuzzy costs and numerical values for uniform, exponential, and normal lead-time demand are compared. Necessary graphical presentations are also given besides numerical illustrations. This model can also be extended taking lead-time demand as fuzzy random variables. 


\section{References}

[1] L. A. Zadeh, "Fuzzy sets," Information and Control, vol. 8, no. 3, pp. 338-353, 1965.

[2] R. E. Bellman and L. A. Zadeh, "Decision-making in a fuzzy environment," Management Science, vol. 17, no. 4, pp. B141-B164, 1970.

[3] H. Tanaka, T. Okuda, and K. Asai, "On fuzzy mathematical programming," Journal of Cybernetics, vol. 3, no. 4, pp. 37-46, 1974.

[4] H. J. Zimmermann, "Description and optimization of fuzzy system," International Journal of General Systems, vol. 2, no. 4, pp. 209-215, 1976.

[5] H. J. Zimmermann, "Fuzzy linear programming with several objective functions," Fuzzy Sets and Systems, vol. 1, pp. 46-55, 1978.

[6] L. Li, S. N. Kabadi, and K. P. K. Nair, "Fuzzy models for single-period inventory problem," Fuzzy Sets and Systems, vol. 132, no. 3, pp. 273-289, 2002.

[7] M. O. Abuo-El-Ata, H. A. Fergany, and M. F. El-Wakeel, "Probabilistic multi-item inventory model with varying order cost under two restrictions: a geometric programming approach," International Journal of Production Economics, vol. 83, no. 3, pp. 223-231, 2003.

[8] C. Kao and W.-K. Hsu, "A single-period inventory model with fuzzy demand," Computers $\mathcal{E}$ Mathematics with Applications, vol. 43, no. 6-7, pp. 841-848, 2002.

[9] H. A. Fergany and M. F. El-Wakeel, "Probabilistic single-item inventory problem with varying order cost under two linear constraints," Journal of the Egyptian Mathematical Society, vol. 12, no. 1, pp. 71-81, 2004.

[10] F. Raafat, "Survey of literature on continuously deteriorating inventory models," Journal of the Operational Research Society, vol. 42, no. 1, pp. 27-37, 1991.

[11] A. F. Hala and M. E. EI-Saadani, "Constrained single period stochastic uniform inventory model with continuous distributions of demand and varying holding cost," Journal of Mathematics and Statistics, vol. 2, no. 1, pp. 334-338, 2006.

[12] H. Katagiri and H. Ishii, "Some inventory problems with fuzzy shortage cost," Fuzzy Sets and Systems, vol. 111, no. 1, pp. 87-97, 2000.

[13] I. Moon and S. Choi, "A note on lead time and distributional assumptions in continuous review inventory models," Computers $\mathcal{E}$ Operations Research, vol. 25, no. 11, pp. 1007-1012, 1998.

[14] Y. J. Lai and C. L. Hwang, Fuzzy Mathematical Programming: Methods and Applications, Lecture Notes in Economics and Mathematical Systems, Springer, Heidelberg, Germany, 1992.

[15] Y. J. Lai and C. L. Hwang, Fuzzy Multiple Objective Decision Making, Lecture Notes in Economics and Mathematical Systems, Springer, Berlin, Germany, 1994.

[16] L.-Y. Ouyang and H.-C. Chang, "A minimax distribution free procedure for mixed inventory models involving variable lead time with fuzzy lost sales ," International Journal of Production Economics, vol. 76, no. 1, pp. 1-12, 2002.

[17] G. S. Mahapatra and T. K. Roy, "Fuzzy multi-objective mathematical programming on reliability optimization model," Applied Mathematics and Computation, vol. 174, no. 1, pp. 643-659, 2006.

[18] M. Hariga and M. Ben-Daya, "Some stochastic inventory models with deterministic variable lead time," European Journal of Operational Research, vol. 113, no. 1, pp. 42-51, 1999.

[19] T. K. Roy and M. Maiti, "A fuzzy EOQ model with demand-dependent unit cost under limited storage capacity," European Journal of Operational Research, vol. 99, no. 2, pp. 425-432, 1997.

[20] Y.-S. Zheng, "Optimal control policy for stochastic inventory systems with Markovian discount opportunities," Operations Research, vol. 42, no. 4, pp. 721-738, 1994.

[21] K. T. Atanassov, "Intuitionistic fuzzy sets," Fuzzy Sets and Systems, vol. 20, no. 1, pp. 87-96, 1986.

[22] K. Atanassov and G. Gargov, "Interval valued intuitionistic fuzzy sets," Fuzzy Sets and Systems, vol. 31, no. 3, pp. 343-349, 1989.

[23] K. T. Atanassov, "Open problems in intuitionistic fuzzy sets theory," in Proceedings of the 6th Joint Conference on Information Sciences (JCIS '02), vol. 6, pp. 113-116, Research Triange Park, NC, USA, March 2002.

[24] K. Atanassov and V. Kreinovich, "Intuitionistic fuzzy interpretation of interval data," Notes on Intuitionistic Fuzzy Sets, vol. 5, no. 1, pp. 1-8, 1999.

[25] K. Atanassov, Intuitionistic Fuzzy Sets: Theory and Applications, vol. 35 of Studies in Fuzziness and Soft Computing, Springer Physica, Berlin, Germany, 1999.

[26] P. K. Maji, R. Biswas, and A. R. Roy, "Intuitionistic fuzzy soft sets," The Journal of Fuzzy Mathematics, vol. 9, no. 3, pp. 677-692, 2001. 
[27] M. Nikolova, N. Nikolov, C. Cornelis, and G. Deschrijver, "Survey of the research on intuitionistic fuzzy sets," Advanced Studies in Contemporary Mathematics, vol. 4, no. 2, pp. 127-157, 2002.

[28] S. Rizvi, H. J. Naqvi, and D. Nadeem, "Rough intuitionistic fuzzy sets," in Proceedings of the 6th Joint Conference on Information Sciences (JCIS '02), vol. 6, pp. 101-104, Research Triange Park, NC, USA, March 2002.

[29] P. P. Angelov, “Optimization in an intuitionistic fuzzy environment," Fuzzy Sets and Systems, vol. 86, no. 3, pp. 299-306, 1997.

[30] P. P. Angelov, "Intuitionistic fuzzy optimization," Notes on Intutionistic Fuzzy Sets, vol. 1, pp. 27-33, 1995.

[31] P. P. Angelov, “Intuitionistic fuzzy optimization,” Notes on Intutionistic Fuzzy Sets, vol. 1, no. 2, pp. 123-129, 1995.

[32] P. Pramanik and T. K. Roy, "An intuitionistic fuzzy goal programming approach to vector optimization problem," Notes on Intutionistic Fuzzy Sets, vol. 11, no. 1, pp. 1-14, 2005.

[33] B. Jana and T. K. Roy, "Multi-objective intuitionistic fuzzy linear programming and its application in transportation model," Notes on Intuitionistic Fuzzy Sets, vol. 13, no. 1, pp. 34-51, 2007.

[34] L.-Y. Ouyang and H.-C. Chang, "Mixture inventory model involving setup cost reduction with a service level constraint," Opsearch, vol. 37, no. 4, pp. 327-339, 2000.

[35] M. J. Paknejad, F. Nasri, and J. F. Affisco, "Defective units in a continuous review (s, Q) system," International Journal of Production Research, vol. 33, no. 10, pp. 2767-2777, 1995.

[36] A. Kaufmann and M. Gupta, Fuzzy Mathematical Models in Engineering and Management Science, North Holland, Amsterdam, The Netherlands, 1988. 


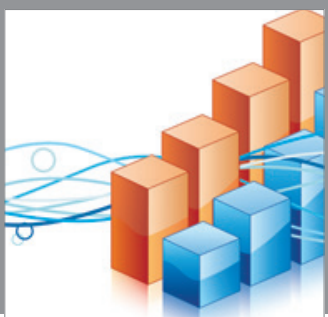

Advances in

Operations Research

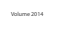

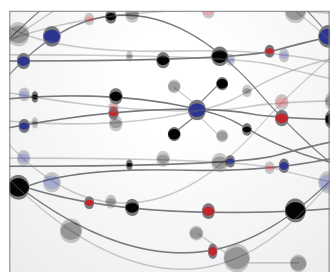

\section{The Scientific} World Journal
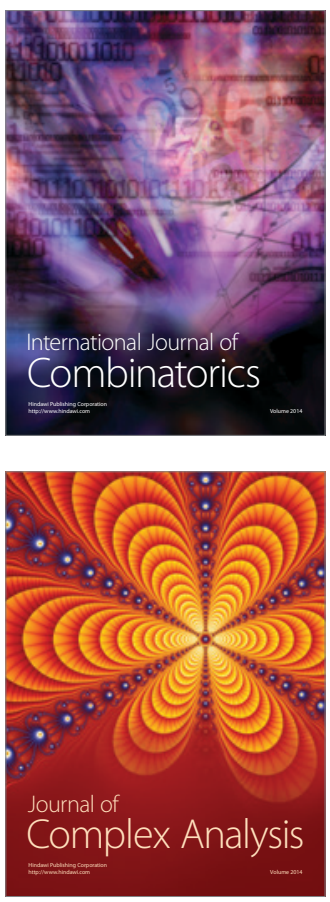

International Journal of

Mathematics and

Mathematical

Sciences
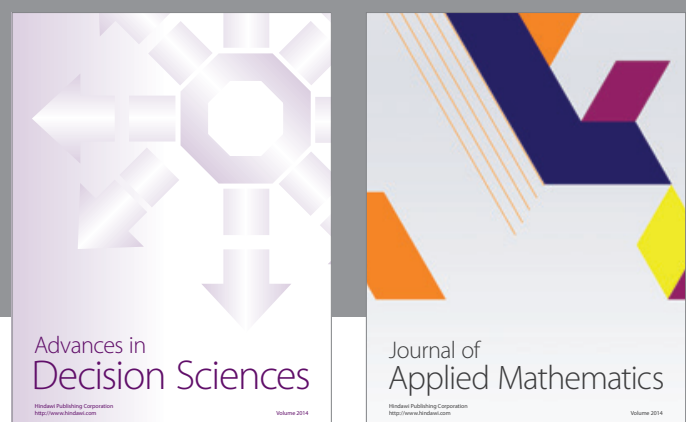

Journal of

Applied Mathematics
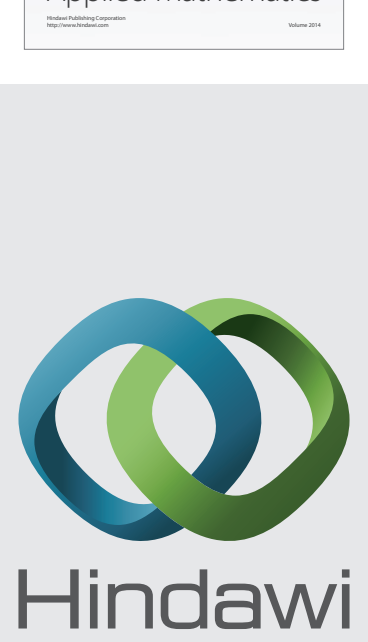

Submit your manuscripts at http://www.hindawi.com
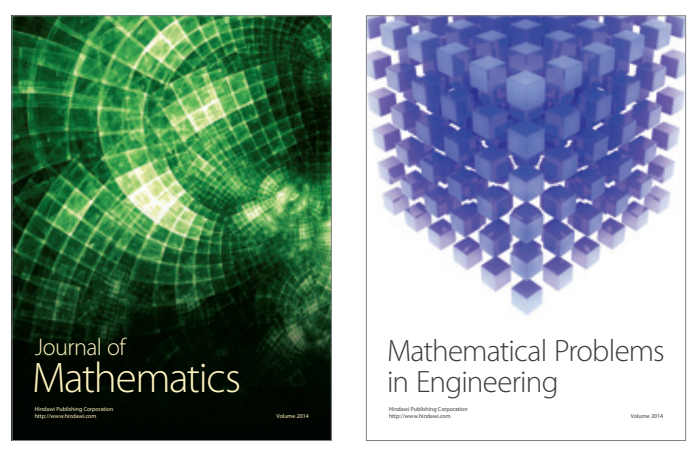

Mathematical Problems in Engineering
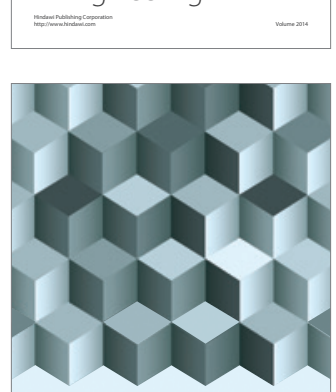

Journal of

Function Spaces
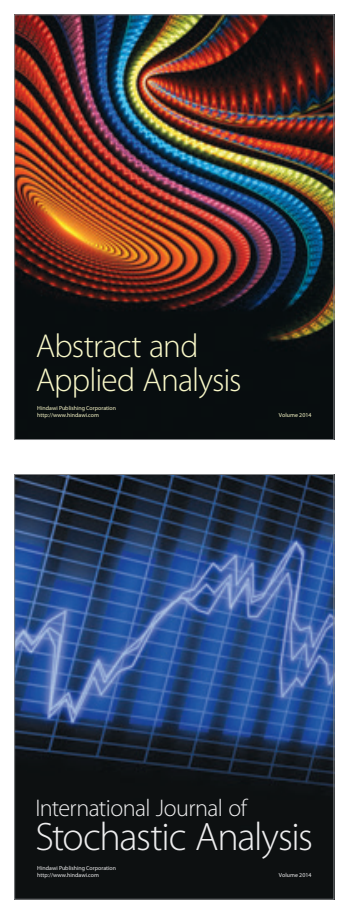

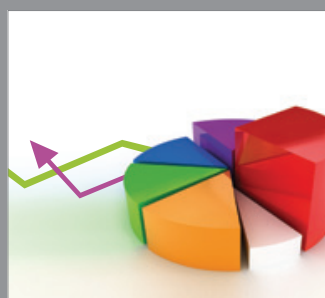

ournal of

Probability and Statistics

Promensencen
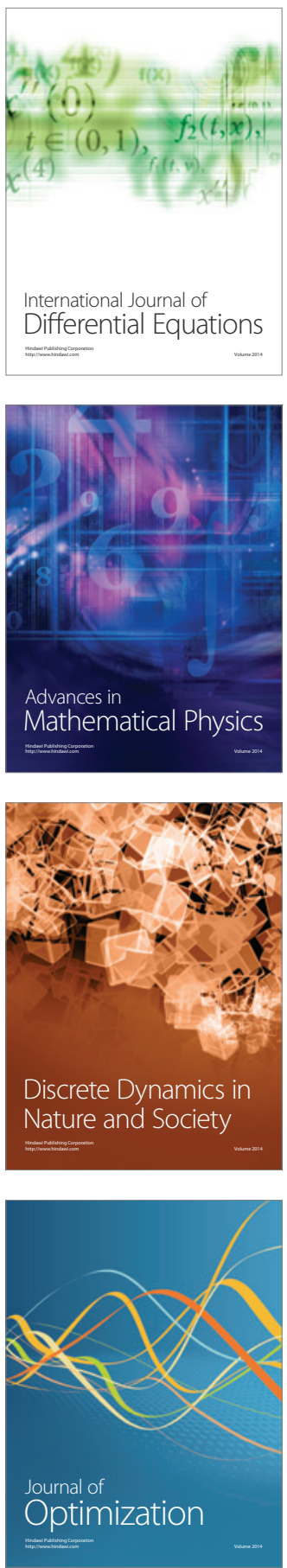